\title{
Intrinsic Strange/Charmed Quarks Inside of a Strangeless/Charmless Hadron.
}

\author{
Ariel Zhitnitsky ${ }^{a}$ \\ Physics and Astronomy Department \\ University of British Columbia \\ Vancouver, BC V6T 1Z1, Canada
}

\begin{abstract}
We discuss few, apparently different, but actually tightly related problems: a)Strangeness in the nucleon; b) $B \rightarrow \eta^{\prime} X$ decays; c) Intrinsic charm in the proton spin problem. We argue that all these problems have a common origin and related to the OZI rule violation in $0^{ \pm}$vacuum channels . It leads to a noticeable role of a nonvalence component with $0^{ \pm}$quantum numbers in a hadron.
\end{abstract}

1. Nowadays it is almost accepted that a nonvalence component in a hadron could be very high, much higher than naively one could expect from the perturbative estimations. Experimentally, such a phenomenon was observed in a number of places. Let me mention only one of them. As is known, the matrix element $\langle N|\bar{s} s| N\rangle$ is not vanishing, as naively one could expect, but rather, is the same order of magnitude as valence matrix element $\langle N|\bar{d} d| N\rangle$. Standard arguments, see e.gl, which lead to this conclusion are based on the analysis of the so-called $\sigma$ term: $\frac{m_{u}+m_{d}}{2}\langle p|\bar{u} u+\bar{d} d| p\rangle=45 \mathrm{MeV}$. Assuming octet-type $S U(3)$ breaking to be responsible for the mass splitting in the baryon octet, we find

$$
\langle p|\bar{u} u| p\rangle \simeq 3.5,\langle p|\bar{d} d| p\rangle \simeq 2.8,\langle p|\bar{s} s| p\rangle \simeq 1.4 .
$$

In spite of the very rough estimations presented above, we believe that a reader is convinced that : a) a magnitude of the nucleon matrix element for $\bar{s} s$ is not small; b) the large value for this matrix element is due to the nontrivial QCD vacuum structure where vacuum expectation values of $u, d, s$ quarks are developed and they have the same order in magnitude: $\langle p|\bar{s} s| p\rangle \sim\langle 0|\bar{s} s| 0\rangle \sim$ $\langle 0|\bar{d} d| 0\rangle$. This result is in a variance with the standard OZI rule expectation predicting that any non-valence matrix element is suppressed in comparison with a similar in structure, but valence one, see refla for details on the QCD explanation of the OZI rule violation. c) The vacuum channels are very special in a sense that the OZI rule in those channels is badly broken while in the vector channel the OZI rule works well.

2. Now we want to discuss a similar example where we believe the OZI rule violation emerges like in eq. (11). Recently CLEO has reported a very

a Talk given at the "HADRON 97". BNL, Brookhaven, August 24-28, 1997. 
large branching ratio for the production of $\eta^{\prime}$ :

$$
\operatorname{Br}\left(B \rightarrow \eta^{\prime}+X\right) \simeq 7.5 \cdot 10^{-4} ; \quad B r\left(B \rightarrow \eta^{\prime}+K\right) \simeq 7.8 \cdot 10^{-5}
$$

To get a feeling of how large these numbers are, we present for comparison the branching ratios for the inclusive and exclusive productions of $J / \psi$ meson $\theta$ :

$$
\operatorname{Br}(B \rightarrow J / \psi(\text { direct })+X) \simeq 8.0 \cdot 10^{-3} ; \quad \operatorname{Br}(B \rightarrow J / \psi+K) \simeq 1 \cdot 10^{-3}
$$

These processes are due to the Cabbibo favored $b \rightarrow \bar{c} c s$ decay which is largest possible amplitude without charmed hadrons (like $D, D_{s}, \Lambda_{c} \ldots$ ) in the final state. The comparison of these two numbers shows that the amplitudes of processes (2) are only by a factor of 3 less than the Cabbibo favored amplitudes $b \rightarrow \bar{c} c s \rightarrow J / \psi s(3)$. It is clear that data (2) is in severe contradiction with a standard view of the process at the quark level as a decay of the $b$-quark into light quarks which could be naively suggested keeping in mind the standard picture of $\eta^{\prime}$ as a $\mathrm{SU}(3)$ singlet meson made of the $u-, d-$ and $s$-quarks. In this picture decay (2) must be proportional to the Cabbibo suppression factor $V_{u b}$, and therefore the standard approach has no chance to explain data (2). Indeed, an assumption that the $\eta^{\prime}$ is made exclusively of light quarks leads to the following ratio for two pseudoscalar particles $\eta^{\prime}$ and $\eta_{c}(1 S)$ :

$$
\frac{\Gamma\left(B \rightarrow \eta^{\prime}+X\right)}{\Gamma\left(B \rightarrow \eta_{c}(1 S)+X\right)} \sim \frac{1}{3}\left(\frac{V_{b u}}{V_{b c}}\right)^{2}\left(\frac{f_{\eta^{\prime}}}{f_{\eta_{c}}}\right)^{2}\left(\frac{\Omega_{b \rightarrow \eta^{\prime}+X}}{\Omega_{b \rightarrow \eta_{c}+X}}\right) \sim 3 \cdot 10^{-4} .
$$

Here $\Omega_{b \rightarrow \eta^{\prime}+X}$ and $\Omega_{b \rightarrow \eta_{c}+X}$ are the corresponding phase volumes for two inclusive decays; $\left(\frac{V_{b u}}{V_{b c}}\right) \simeq 0.08$. The matrix element $\left\langle\eta^{\prime}(p)\left|\bar{u} \gamma_{\mu} \gamma_{5} u\right| 0\right\rangle=\frac{-i}{\sqrt{3}} f_{\eta^{\prime}} p_{\mu} \simeq$ $(0.5 \div 0.8) \frac{-i}{\sqrt{3}} f_{\pi} p_{\mu}$ is known numerically; $f_{\eta_{c}} \simeq 400 \mathrm{MeV}$ can be estimated from the $\eta_{c} \rightarrow \gamma \gamma$ decay. Therefore, the standard mechanism yields a very small contribution in comparison with data (2): $\operatorname{Br}\left(B \rightarrow \eta^{\prime}+X\right) \sim 1.5 \cdot 10^{-6}$. We should mention that the factorization procedure used in the estimate (任) does not work well. A phase factor introduced into this formula is also a rough simplification: in reality, an inclusive spectrum is much more complicated function than a simple factor $\Omega_{b \rightarrow \eta^{\prime}+X}$ obtained as a result of two-particle decay of a colorful heavy quark $b \rightarrow \eta^{\prime}\left(\eta_{c}\right)+d(s)$ instead of the physical $B$ meson. However, it is obvious that all these effects due to a non-factorizability, gluon corrections, as well as $O\left(1 / m_{b}, 1 / N\right)$ terms omitted in (何), cannot substantially change our estimate. We therefore conclude that the image of the $\eta^{\prime}$ meson as the $\mathrm{SU}(3)$ singlet quark state made exclusively of the $u, d, s$ quarks is not adequate to the problem at hand.

3. In view of the failure of the standard approach to the $B \rightarrow \eta^{\prime}+X$ decay which treats the $\eta^{\prime}$ as the $\mathrm{SU}(3)$ singlet quark state made exclusively of the 
$u, d, s$ quarks, we suggest an alternative mechanism for the $B \rightarrow z^{\prime}+X$ decay which is specific to the uniqueness of the $\eta^{\prime}$. It has been known ${ }^{6}$, that the $\eta^{\prime}$ is a messenger between two worlds: the world of light hadrons and a less studied world of gluonia. In other words, it is a very special meson strongly coupled to gluons. We suggest the following picture for the process of interest: the $b \rightarrow c \bar{c} s$ decay is followed by the conversion of the $c \bar{c}$-pair into the $\eta^{\prime}$. This means that the matrix element $\left\langle 0\left|\bar{c} \gamma_{\mu} \gamma_{5} c\right| \eta^{\prime}(p)\right\rangle=i f_{\eta^{\prime}}^{(c)} p_{\mu}$ is not zero due to the $c \bar{c} \rightarrow$ gluons transition:

$$
f_{\eta^{\prime}}^{(c)}=-\frac{1}{16 \pi^{2} m_{\eta^{\prime}}^{2}} \frac{1}{m_{c}^{2}}\left\langle 0\left|g^{3} f^{a b c} G_{\mu \nu}^{a} \tilde{G}_{\nu \alpha}^{b} G_{\alpha \mu}^{c}\right| \eta^{\prime}\right\rangle+0\left(1 / m_{c}^{4}\right)+\ldots
$$

Of course, since one deals here with virtual c-quarks, this matrix element is suppressed by the $1 / m_{c}^{2}$. However, the c-quark is not very heavy, and the suppression $1 / m_{c}^{2}$ is not large numerically. At the same time, the Cabbibo enhancement of the $b \rightarrow c$ transition in comparison to $b \rightarrow u$ is a much more important factor which makes this mechanism work. One can estimate $f_{\eta^{\prime}}^{(c)}=(50 \sim 180) \mathrm{MeV}$ indirectly Gusing a combination of the OPE, large $N$ approach and QCD low energy theorems. If one assumes the saturation of the experimental data (2) by suggested mechanism one obtains $f_{\eta^{\prime}}^{(c)}(\exp .) \simeq 140 \mathrm{MeV}$, sed for details.

4. Here we want to calculate $f_{\eta^{\prime}}^{(c)}$ directly using the Interacting Instanton Liquid Model (IILM), see for a review. The calculation is based on the numerical evaluation of the following two-point Euclidean correlation functions

$$
K_{22}(x)=\left\langle 0\left|T g^{2} G_{\mu \nu}^{a} \tilde{G_{\mu \nu}^{a}}(x), \quad g^{2} G_{\mu \nu}^{a} \tilde{G}_{\mu \nu}^{a}(0)\right| 0\right\rangle
$$

and similar for $K_{23}(x)$ and $K_{33}(x)$ where two-gluon operator $g^{2} G_{\mu \nu}^{a} \tilde{G_{\mu \nu}^{a}}$ is replaced by the three- gluon operator $g^{3} f^{a b c} G_{\mu \nu}^{a} \tilde{G}_{\nu \lambda}^{b} G_{\lambda \mu}^{c}(x)$ once (for $K_{23}(x)$ ) or twice (for $K_{33}(x)$ ) correspondingly. The magnitude $f_{\eta^{\prime}}^{(c)}$ can be obtained from the calculation of the correlation functions:

$$
\left|\frac{f_{\eta^{\prime}}^{(c)} \sqrt{3} m_{c}^{2}}{f_{\eta^{\prime}}}\right|=\left|\frac{K_{23}(x \rightarrow \infty)}{K_{22}(x \rightarrow \infty)}\right|=\sqrt{\frac{K_{33}(x \rightarrow \infty)}{K_{22}(x \rightarrow \infty)}}
$$

The corresponding measurements of $K_{23}, K_{33}$ both ratios entering (7) has been carrying out in ref. 3 where it was found the stabilization at large enough $\mathrm{x}>0.8 \mathrm{fm}$ at the same numerical value. We take it as an indication that $\eta^{\prime}$ contribution does in fact dominate in this region. Final result of this calculations can be presented in the form 9 :

$$
\left|f_{\eta^{\prime}}^{(c)} / f_{\eta^{\prime}}\right| \simeq(0.85 \sim 1.22)
$$


The obtained result is in a fair agreement with "experimental" value $f_{\eta^{\prime}}^{(c)}(\exp .) \simeq$ $140 \mathrm{MeV}$ needed to explain CLEO measurements, inside the uncertainties.

5. The next logical question to ask is whether the connection between strong instanton fields and charm lead to phenomena unrelated $t \rho \eta^{\prime}$. One intriguing direction is to study the "intrinsic charm" (see e.g. 10) of other hadrons. In particular, one could consider the charm contribution to the spin of the nucleon 11 . The relevant matrix element is the axial current of the charmed quark, $\left\langle N\left|\bar{c} \gamma_{\mu} \gamma_{5} c\right| N\right\rangle=g_{A}^{(c)} \bar{N} \gamma_{\mu} \gamma_{5} N$. It could be generated e.g. by the $\eta^{\prime}$ "cloud" of the nucleon. Assuming now the $\eta^{\prime}$ dominance in this matrix element one could get the following Goldberger-Treiman type relation 11 $g_{A}^{(c)}=\frac{1}{2 M_{N}} g_{\eta^{\prime} N N} f_{\eta^{\prime}}^{(c)}$. Although the value of $g_{\eta^{\prime} N N}$ is unknown, and its phenomenological estimates vary significantly $g_{\eta^{\prime} N N}=3-7$, one gets from this estimate a surprisingly large contribution $\left\langle N\left|\bar{c} \gamma_{\mu} \gamma_{5} c\right| N\right\rangle=(0 . .2 \sim 0.5) \bar{N} \gamma_{\mu} \gamma_{5} N$ comparable to the light quark one, se1211! Ultimately, the contribution of the charmed quarks in polarized deep-inelastic scattering may be tested experimentally, by tagging the charmed quark jets (e.g. by COMPASS experiment at CERN).

6. The situation reminds me the $J / \psi$ discovery in 1974 , when a charmonium state ("hidden charm") was observed simultaneously in $e^{+} e^{-}$collisions at SLAC and at the proton machine at Brookhaven. I believe that we are now facing a similar case, when different experimental groups see the "intrinsic charm" in polarized DIS and in B-decays (2) simultaneously, se 11 for details.

1. J.F.Donoghue, E.Golowich and B.R.Holstein, Dynamics of the Standard Model, Cambridge University Press, 1992.

2. A.R. Zhitnitsky, Phys. Rev. D55 (1997) 3006; Phys. Lett.B404 (1997), 328; Nucl. Phys. B, Proc. Suppl.55A (1997) 40.

3. P. Kim [CLEO], Talk at FCNC 1997, Santa Monica, CA (Feb 1997)

F. Würthwein, hep-ex/9706010

4. Particle Data Group, Phys. Rev. D54 (1996) 1.

5. E. Witten, Nucl. Phys. B156 (1979) 269.

6. G. Veneziano, Nucl. Phys. B159 (1979) 213.

7. I. Halperin and A. Zhitnitsky, Phys. Rev. D56 (1997) 7247; Phys. Rev.Lett. 80 (1998) 438.

8. T. Schäfer and E. V. Shuryak, hep-ph/9610451, Rev.Mod.Phys.,in press.

9. E. V. Shuryak and A. Zhitnitsky, Phys. Rev. D57 (1998) 2001.

10. S. J. Brodsky, P.Hoyer, A.H.Mueller and W.-K.Tang, Nucl.Phys. B369 (1992) 519; S. J. Brodsky, hep-ph/9503391.

11. I. Halperin and A. Zhitnitsky, hep-ph/9706251.

12. J.H. Kühn and V.I. Zakharov, Phys. Lett. B252 (1990) 615. 